\title{
RPE epithelial-mesenchymal transition plays a critical role in the pathogenesis of proliferative vitreoretinopathy
}

\author{
Xiaohua $\mathrm{Li}^{1,2,3,4}$, Mingwei Zhao ${ }^{5}$, Shikun $\mathrm{He}^{5,6}$ \\ ${ }^{1}$ Department of Ophthalmology, Henan Provincial People's Hospital, Zhengzhou 450003, China; ${ }^{2}$ Henan Eye Hospital, Henan Eye Institute, Henan \\ Key Laboratory of Ophthalmology and Visual Science, Zhengzhou 450003, China; ${ }^{3}$ Department of Ophthalmology, People's Hospital of Zhengzhou \\ University, Zhengzhou 450003, China; ${ }^{4}$ People's Hospital of Henan University, Zhengzhou 450003, China; ${ }^{5}$ Ophthalmology Optometry Center, \\ Peking University People's Hospital, Beijing Key Laboratory of Diagnosis and Therapy of Retinal and Choroid Diseases, Beijing 100044, China; \\ ${ }^{6}$ Departments of Pathology and Ophthalmology, Keck School of Medicine of the University of Southern California, Los Angeles, CA, USA \\ Correspondence to: Shikun He, MD. Departments of Pathology and Ophthalmology, Keck School of Medicine of the University of Southern \\ California, 2011 Zonal Ave, Los Angeles, CA 90033, USA. Email: shikunhe@usc.edu; Mingwei Zhao, MD, PhD. Ophthalmology Optometry Center, \\ Peking University People's Hospital, Beijing Key Laboratory of Diagnosis and Therapy of Retinal and Choroid Diseases, \#11 Xizhimen South \\ Street, Beijing 100044, China. Email: dr_zhaomingwei@163.com. \\ Provenance and Peer Review: This article was commissioned by the Editorial Office, Annals of Translational Medicine. The article did not undergo \\ external peer review. \\ Comment on: Cui L, Yu Y, Jin X, et al. miR-194 suppresses epithelial-mesenchymal transition of retinal pigment epithelial cells by directly targeting \\ ZEB1. Ann Transl Med 2019;7:751.
}

Submitted Jan 21, 2020. Accepted for publication Feb 18, 2020.

doi: $10.21037 / \mathrm{atm} .2020 .03 .86$

View this article at: http://dx.doi.org/10.21037/atm.2020.03.86

Serious proliferative vitreoretinopathy (PVR) may lead to vision loss or blindness due to the generation of PVR membrane in vitreous and retina and tractional retinal detachment, it is often seen after retinal reattachment surgery or retinal wound. RPE epithelial-mesenchymal transition (EMT) is a critical step of wound healing response in $\operatorname{PVR}(1,2)$.

The transition of a cell from epithelial phenotype to mesenchymal-like cell in certain conditions is called EMT. Three types of EMT have been recognized (3), type 1 EMT functions for tissue and embryo development, type 2 EMT often refers to the response of tissue wound and scar formation, type 3 EMT involves in the process of tumor invasion.

There are numbers of well-recognized changes in the process of EMT including but not limited to the aberrant expression of cell surface molecules, cytoskeletal reorganization, loss of the junctions between cells and apical-basal polarity, extracellular matrix over production, increased cell proliferation and migration (4-6). In order to confirm the development of EMT, at least the following changes of cellular contents and function during EMT should be closely monitored: notably, EMT is dynamic and reversible, therefore, the reverse process is named as mesenchymal-epithelial transition (MET), suggesting that under certain conditions, some transdifferentiated epithelial cell may be converted to epithelial cell and thus the manipulation of phenotype changes between epithelial and mesenchymal cell is possible (Figure 1A) (4-6).

\section{Cellular changes during EMT}

Lost or decreased:

* Epithelial adhesion molecular: E-cadherin, Occludin;

* Apical-basal polarity.

Acquired:

* $\alpha$-smooth muscle actin ( $\alpha$-SMA);

* Intermediate filament protein: Vimentin;

- Migration and proliferation.

Intracellular pathways:

* Smad2/3;

* Smad7.

Transcriptional factors:

* Snail; 


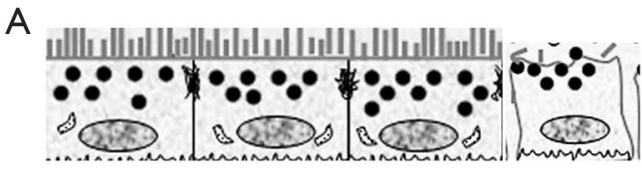

Epithelium

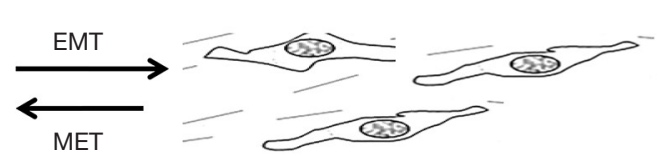

Mesenchymal cell

B

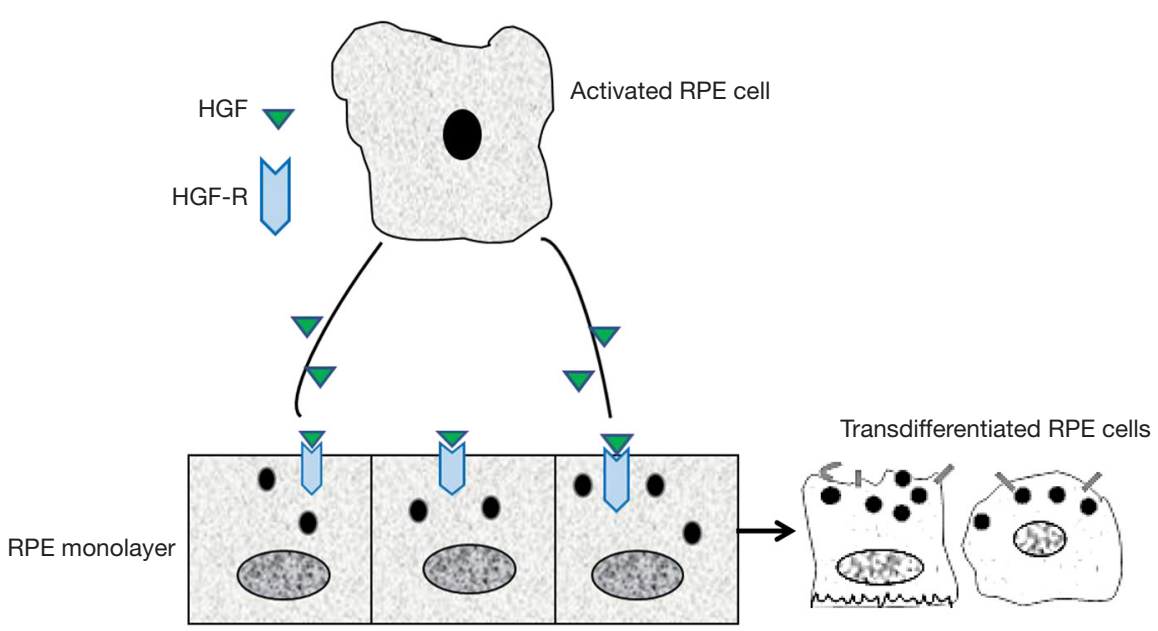

Figure 1 The diagram of transition between EMT and MET. (A) At the specific conditions, the switch between EMT and MET will occur; (B) activated RPE cell (by inflammatory factors) release HGF and HGF induces the disconnection of individual RPE from the monolayer to dedifferentiated RPE through a transmembrane tyrosine kinase receptor (HGF receptor). EMT, epithelial-mesenchymal transition; MET, mesenchymal-epithelial transition.

* Peroxisome proliferator-activated receptor- $\gamma$ (PPAR- $\gamma$ ).

We should realize that EMT is regulated by numerous factors including inflammatory cytokines and growth factors (TGF- $\beta$, CTGF, PDGF) (7), and extracellular matrix (ECM) has also been found to stimulate extracellular signals that induce EMT. $\alpha$-SMA is a marker of EMT, among growth factors, the strongest inducer of $\alpha$-SMA is TGF- $\beta$ in transdifferentiated cells, at the time of the development of EMT, the expression of TGF- $\beta$ is upregulated, while the EMT inhibitor factors including Smad7, IкB $\alpha$ and PPAR- $\gamma$ are suppressed (8).

It is well recognized that the RPE cell is the major cell to participate in the formation of EMT and PVR, this notion is supported by the studies of numbers of fibrotic retinal diseases such as neovascular age-related macular degeneration (nAMD), retinal fibrosis complicated by diabetes, PVR and choriocapillaris atrophy (9).

We have to address that in normal eye, RPE cells are non-migratory as well as non-proliferative, however, the ability of the RPE transdifferentiation is greatly elevated from its original essence in vitro after activation. A major feature of RPE cell EMT is the upregulation of $\alpha$-SMA, and the majority of the transdifferentiated cell in PVR membrane is RPE cell which produce the traction force and induce retinal detachment. The abnormal of the RPE behavior can be demonstrated in retinal wound healing in vivo. The EMT phenotype of RPE cell has been induced in vitro which is associated with up-regulation of $\alpha$-SMA expression and loss of the epithelial polarity and junction proteins. After transdifferentiation, the RPE cell behaves like macrophage to migrate, like muscle cell to contract, like immune proponent cell to produce more cytokines and growth factors.

As mentioned above, RPE cell transdifferentiation could be a predisposing step in the development of PVR, however, the fundamental mechanisms of the RPE cell EMT is remained unclear. In previous study, we believe that HGF is the major mediator of EMT (cell migration, proliferation 
and increased EMT markers) (10), because treatment of the RPE monolayer with HGF results in RPE cell disconnecting from each other at the existing of TNF $\alpha$ and PDGF and stimulates the migration and proliferation of the separated RPE cells, and therefore a PVR membrane is formed with the enrichment of cellular components (Figure $1 B$ ). HGF receptor (C-Met) plays an essential role in the induction of such kind of function by ligation with HGF. One of important factor we have to mention is that CTGF is the major mediator of EMT and retinal fibrosis (11). CTGF works as a downstream gene of TGF- $\beta$, takes action on EMT and fibrosis by stimulating cell proliferation and ECM deposition. Importantly, CTGF can induce rabbit PVR when injected into the vitreous cavity. Although many fibrogenic growth factors/cytokines produced by injured tissues participate in the process of EMT, TGF- $\beta$ is believed to play a central role. Up-regulation of other pro-fibrotic cytokines and ECM genes mediated by TGF- $\beta$ prime the microenvironment favorable for fibrosis. Treatment of cultured RPE cells with TGF- $\beta$ induces RPE cell transdifferentiation into fibroblast-like cell through EMT. Although PDGF and CTGF are well known to be expressed in human PVR membranes, the downstream signaling of those growth factors is believed to target on TGF- $\beta$, suggesting that TGF- $\beta$ could orchestrate the secondary effects of other stimulators on EMT in PVR.

In term of EMT signaling, Smad2/3 has been demonstrated to be the key signaling pathways of EMT induced by TGF- $\beta$, inhibition of TGF- $\beta$ via $\operatorname{Smad} 2 / 3$ signaling has been demonstrated to suppress PVR in an experimental PVR model by knocking down Smad2/3, blocking the Smad2/3 signaling also inhibits the expression of $\alpha$-SMA, the EMT marker, and collagen IV in RPE cell, while the same effect can be observed by the presence of Smad7. The results suggest that the $\mathrm{Smad} 2 / 3$ signaling is required in the process of TGF- $\beta$ induced EMT. Besides Smad2/3 signaling, the involvement of other signaling pathway (12), such as $\beta$-catenin/Wnt and Hippo signaling $(2,13)$, PKC pathway, NOTCH signaling, YAP1-snail1 axis (14), ERK and AKT in the induction of EMT should also be concerned about.

The critical marker of epithelial cell transdifferentiation is the increased expression of $\alpha$-SMA. Transdifferentiation of RPE cells to $\alpha$-SMA-positive phenotype is considered to be an example of epithelial-transdifferentiation. $\alpha$-SMA expression in transdifferentiated cells is induced by TGF- $\beta$. Further, the EMT process in human fetal RPE cells is highly regulated by TGF- $\beta 2$ and ECM proteins. It was reported that the expressions of TGF- $\beta$ and TGF- $\beta$ R 2 are detected in PVR membranes, so the role of the TGF- $\beta$ signaling in the regulation of EMT should be addressed.

TGF $-\beta$ signaling promotes EMT by activating several transcription factors which include Twist and most significantly, Snail1/2 (15). The activation of these transcription factors suppresses the epithelial phenotype and transdifferentiation of epithelial cells into motile cell type. Snail is an immediate-early response gene of TGF- $\beta$, it could be activated through the signaling of $S \operatorname{mad} 2 / 3$ and Smad4. More importantly, inhibition of Snail expression is able to block TGF- $\beta$-induced $\alpha$-SMA expression in lens epithelial cells. This implies that Snail plays an essential role in promoting the ability of epithelial cell transdifferentiation.

In consideration of the roles of epigenetic factors (DNA methylation, histone modification and non-coding RNA) in the pathogenesis of EMT, we suggest that MeCP2 is an important mediator in the induction of EMT and the generation of fibrosis $(16,17)$.

We thought that the pathogenesis of EMT and PVR in RPE cells is strictly regulated by MeCP2 (16). The notion of the importance of MeCP2 in the pathogenesis of PVR is supported by the following evidences: the abundant expression of $\mathrm{MeCP} 2$ and the double labeling of MeCP2 with cytokeratin and $\alpha$-SMA are found in human PVR membrane (16); TGF- $\beta R 2$ and $\operatorname{Smad} 2 / 3$ activation and TGF- $\beta$ induced increase of $\alpha$-SMA and fibronectin are suppressed by MeCP2 siRNA in cultured RPE cells (16); $\mathrm{MeCP} 2$ regulates the expression of $\alpha$-SMA through the signaling pathway of Ras GTPase activating protein (RASAL1) (16). Interestingly, 5-Aza-2'deoxycytidine (a DNA methylation inhibitor) could upregulate the RASAL1 mRNA expression (16). More importantly, the upregulation of PPAR- $\gamma$ is related to the decrease of MeCP2 expression after exposure to 5-AZA. These observations support that $\mathrm{MeCP} 2$ plays a critical role in the induction of EMT, and the development of PVR may be mediated by epigenetic factors (Figure 2). MeCP2 may be the target of PVR treatment.

Besides DNA methylation, EMT and fibrosis are also regulated by histone acetylation/deacetylation (18). So far as best of our knowledge, the regulation of EMT and fibrosis by histone modification has not been reported in RPE, however, previous studies show that the pathological process of EMT and fibrosis are highly regulated by 


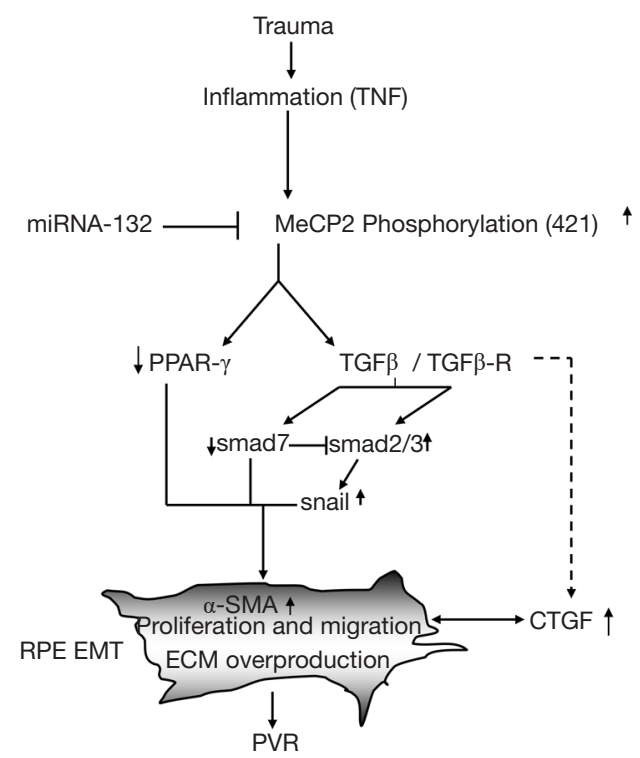

Figure 2 The diagram of MeCP2 in the induction of EMT and PVR formation. Retinal trauma cause inflammation response and up-regulate the expression of methyl-CpG-binding proteins (MeCP2) and its phosphorylation, MeCP2 then activate TGF- $\beta$ and silence another set of genes for example PPAR- $\gamma$ and Smad 7. Meanwhile miR-132 is down-regulated by inflammatory factors, which is a upstream mediator of MeCP2. TGF- $\beta$ activates Smad 2/3, Snail-1 and its down-steam gene CTGF and results in the increasing expression of $\alpha$-SMA and ECM overproduction and $\mathrm{RPE}$ behavior changes such as migration and proliferation. MeCP2 likely plays a central role in regulating EMT and the pathogenesis of PVR. EMT, epithelial-mesenchymal transition; MET, mesenchymal-epithelial transition; ECM, extracellular matrix.

histone modification in certainly systemic diseases including heart, renal, lung and liver fibrosis. Inhibition of HDAC suppressed TGF- $\beta$ induced kidney fibrosis. TSA (a HDAC inhibitor) decreased growth factor induced fibroblast proliferation. Interestingly, the transdifferentiation of corneal stromal cell induced by TGF- $\beta$ is suppressed using TSA. Importantly, in the laser-induced model of CNV, systemic administration of TSA significantly reduces the expression of vascular endothelial growth factor (VEGF), and $\alpha$-SMA in CNV lesions (19). Recently, we demonstrated that intravitreal injection of resveratrol (a SIRT1 activator) not only suppresses TGF- $\beta 2$-induced EMT of RPE cells by deacetylating Smad4, but also induces MET (20), suggesting a possibility to reverse EMT.
One of an important epigenetic factor in the regulation of EMT is non-coding RNA. Recently, roughly 1,300 short RNA sequencing datasets from 13 distinct human tissues have been documented; 3,707 novel mature miRNAs arising from 3,494 novel precursors; according to the current estimates, human genome likely has over 25,000 miRNAs. Many miRNAs might also participate in the regulation of EMT process (21), miRNA-132 may inhibit EMT through suppression of MeCP2 (Figure 2), thus, those miRNAs might also be EMT biomarkers. The importance of miRNA in the pathogenesis of EMT especially in the regulation of PVR has been further addressed by Cui et al. (22), the authors showed that the expression of miR-194 is much stronger in RPE than other retinal layers, knock down miR194 compromised EMT phenotype induced by TGF- $\beta$ in RPE cells, most importantly, intravitreal injection of miR194 is able to inhibit experimental PVR in a rat model, therefore, miR-194 could be a potentially novel therapeutic approach in the treatment of PVR (22).

The mechanisms of EMT and fibrosis formation remain largely under investigation, especially the regulation of EMT by epigenetic factors. More extensive research is needed to clarify the outline of DNA methylation, histone modification, non-coding RNA and RNA methylation mediated genes expression in the initiation of EMT and fibrosis, specifically, the regulation of the expression of $\mathrm{NF}-\kappa \mathrm{B}$, Snail1 and Twist by epigenetic factors in the development of RPE cell EMT and how the RPE cell EMT could be reversed to MET by increasing the MET inducer factors such as BMP7, OCT4, SOX2 and PGC$1 \alpha$ expression which is an important factor in maintaining normal function and phenotype of RPE cell (23). Highlight the mysteriousness behind of EMT is not only important for further understanding of the mechanism of EMT initiation, progression, regression and the switch between EMT and MET through the analysis of transcriptome, proteomic, metabonomics, and epigenome, but also is critical for the potential pharmacologic approach for the treatment of PVR and other fibrotic retinal diseases (24).

\section{Acknowledgments}

Funding: This work was supported by the grants from the National Nature Science Foundation of China (81770952, 81873681, 81770943), the Nature Science Foundation of Henan Province (162300410296) and the "23456" project of Henan Provincial People's Hospital (ZC23456135). 


\section{Footnote}

Conflicts of Interest: The authors have no conflicts of interest to declare.

Ethical Statement: The authors are accountable for all aspects of the work in ensuring that questions related to the accuracy or integrity of any part of the work are appropriately investigated and resolved.

Open Access Statement: This is an Open Access article distributed in accordance with the Creative Commons Attribution-NonCommercial-NoDerivs 4.0 International License (CC BY-NC-ND 4.0), which permits the noncommercial replication and distribution of the article with the strict proviso that no changes or edits are made and the original work is properly cited (including links to both the formal publication through the relevant DOI and the license). See: https://creativecommons.org/licenses/by-nc-nd/4.0/.

\section{References}

1. Casaroli-Marano RP, Pagan R, Vilaró S. Epithelial-mesenchymal transition in proliferative vitreoretinopathy: intermediate filament protein expression in retinal pigment epithelial cells. Invest Ophthalmol Vis Sci 1999;40:2062-72.

2. Tamiya S, KaplanHJ. Role of epithelial-mesenchymal transition in proliferative vitreoretinopathy. Exp Eye Res 2016;142:26-31.

3. Dongre A, Weinberg RA. New insights into the mechanisms of epithelial-mesenchymal transition and implications for cancer. Nat Rev Mol Cell Biol 2019;20:69-84.

4. Persa OD, Niessen CM. Epithelial polarity limits EMT. Nat Cell Biol 2019;21:299-300.

5. Brabletz T. EMT and MET in Metastasis: Where Are the Cancer Stem Cells? Cancer Cell 2012;22:699-701.

6. Pei D, Shu X, Gassama-Diagne A, et al. Mesenchymalepithelial transition in the development and reprogramming. Nat Cell Biol 2019;21:44-53.

7. Yang $\mathrm{S}, \mathrm{Li} \mathrm{H}, \mathrm{Li}$ M, et al. Mechanisms of epithelialmesenchymal transition in proliferative vitreoretinopathy. Discov Med 2015;20:207-17.

8. Wang L, Lu W, Gao YH, et al. Anluohuaxianwan Alleviates Carbon Tetrachloride-Induced Hepatic Fibrosis in Rats through Upregulation of Peroxisome ProliferatorActivated Receptor-Gamma and Downregulation of Nuclear Factor-Kappa B/IкB $\alpha$ Signaling Pathway. World J Tradit Chin Med 2019;5:95-103.

9. Ohlmann A, Scholz M, Koch M, et al. Epithelialmesenchymal transition of the retinal pigment epithelium causes choriocapillaris atrophy. Histochem Cell Biol 2016;146:769-80.

10. Jin M, Barron E, He S, et al. Regulation of RPE intercellular junction integrity and function by hepatocyte growth factor. Invest Ophthalmol Vis Sci 2002;43:2782-90.

11. He S, Chen Y, Khankan R, et al. Connective tissue growth factor as a mediator of intraocular fibrosis. Invest Ophthalmol Vis Sci 2008;49:4078-88.

12. Zhou $X$, Kuang $X$, Long $C$, et al. Curcumin Inhibits Proliferation and Epithelial-Mesenchymal Transition of Retinal Pigment Epithelial Cells Via Multiple Pathways. Curr Mol Med 2017;17:312-9.

13. Kim WK, Kwon $Y$, Jang $M$, et al. $\beta$-catenin activation down-regulates cell-cell junction-related genes and induces epithelial-to-mesenchymal transition in colorectal cancers. Sci Rep 2019;9:18440.

14. Yu S, Zhang Y, Li Q, et al. CLDN6 promotes tumor progression through the YAP1-snail1 axis in gastric cancer. Cell Death Dis 2019;10:949.

15. Manshouri R, Coyaud E, Kundu ST, et al. ZEB1/NuRD complex suppresses TBC1D2b to stimulate E-cadherin internalization and promote metastasis in lung cancer. Nat Commun 2019;10:5125.

16. He S, Barron $\mathrm{E}$, Ishikawa $\mathrm{K}$, et al. Inhibition of DNA methylation and methyl-CpG-binding protein 2 suppresses RPE transdifferentiation: relevance to proliferative vitreoretinopathy. Invest Ophthalmol Vis Sci 2015;56:5579-89.

17. Mann J, Chu DC, Maxwell A, et al. MeCP2 controls an epigenetic pathway that promotes myofibroblast transdifferentiation and fibrosis. Gastroenterology 2010;138:705-14.

18. Noh H, Oh EY, Seo JY, et al. Histone deacetylase-2 is a key regulator of diabetes- and transforming growth factor$\beta 1$-induced renal injury. Am J Physiol Renal Physiol 2009;297:F729-39.

19. Chan N, He S, Spee CK, et al. Attenuation of choroidal neovascularization by histone deacetylase inhibitor. PLoS One 2015;10:e0120587. 
20. Ishikawa $\mathrm{K}, \mathrm{He} \mathrm{S}$, Terasaki H, et al. Resveratrol inhibits epithelial-mesenchymal transition of retinal pigment epithelium and development of proliferative vitreoretinopathy. Sci Rep 2015;5:16386.

21. Chen X, Ye S, Xiao W, et al. Differentially expressed microRNAs in TGF $\beta 2$-induced epithelial-mesenchymal transition in retinal pigment epithelium cells. Int $\mathrm{J}$ Mol Med 2014;33:1195-200.

22. Cui L, Yu Y, Jin X, et al. miR-194 suppresses epithelialmesenchymal transition of retinal pigment epithelial cells

Cite this article as: $\mathrm{Li} \mathrm{X}$, Zhao M, He S. RPE epithelialmesenchymal transition plays a critical role in the pathogenesis of proliferative vitreoretinopathy. Ann Transl Med 2020;8(6):263. doi: $10.21037 /$ atm.2020.03.86 by directly targeting ZEB1. Ann Transl Med 2019;7:751.

23. RosalesMAB, Shu DY, Iacovelli J, et al. Loss of PGC-1 $\alpha$ in $\mathrm{RPE}$ induces mesenchymal transition and promotes retinal degeneration. Life Sci Alliance 2019. doi: 10.26508/ 1sa.201900436.

24. Moon K, Lee HG, Baek WK, et al. Bortezomib inhibits proliferation, migration, and TGF- $\beta 1$-induced epithelial-mesenchymal transition of RPE cells. Mol Vis 2017;23:1029-38. 